\title{
EVALUATION OF VOCAL ACOUSTIC AND EFFICIENCY ANALYSIS PARAMETERS IN MEDICAL STUDENTS AND ACADEMIC TEACHERS WITH USE OF IRIS AND DIAGNOSCOPE SPECIALIST SOFTWARE
}

\author{
HANNA ZIELIŃSKA-BLIŹNIEWSKA ${ }^{1}$, WIESŁAW J. SUŁKOWSKI ${ }^{1,2}$, PIOTR PIETKIEWICZ1, \\ JAROSŁAW MIŁOŃSKI ${ }^{1}$, AGNIESZKA MAZUREK ${ }^{1}$, and JUREK OLSZEWSKI ${ }^{1}$
}

${ }^{1}$ Medical University of Łódź, Łódź, Poland

Department of Otolaryngology and Laryngological Oncology

${ }^{2}$ Nofer Institute of Occupational Medicine, Łódź, Poland

Department of Occupational Diseases and Toxicology

\begin{abstract}
Objectives: The aim of this study was to compare the parameters of vocal acoustic and vocal efficiency analyses in medical students and academic teachers with use of the IRIS and DiagnoScope Specialist software and to evaluate their usefulness in prevention and certification of occupational disease. Material and Methods: The study group comprised 40 women, including students and employees of the Military Medical Faculty, Medical University of Łódź. After informed consent had been obtained from the participant women, the primary medical history was taken, videolaryngoscopic and stroboscopic examinations were performed and diagnostic vocal acoustic analysis was carried out with the use of the IRIS and DiagnoScope Specialist software. Results: Based on the results of the performed measurements, the statistical analysis evidenced the compatibility between two software programs, IRIS and DiagnoScope Specialist, with the only exception of the F4 formant. The mean values of vocal acoustic parameters in medical students and academic teachers, obtained by means of the IRIS software, can be used as standards for the female population not yet developed by the producer. When using the DiagnoScope Specialist software, some mean values were higher and some lower than the standards specified by the producer. Conclusions: The study evidenced the compatibility between two measurement software programs, IRIS and DiagnoScope Specialist, except for the F4 formant. It should be noted that the later has advantage over the former since the standard values of vocal acoustic parameters have been worked out by the producer. Moreover, they only slightly departed from the values obtained in our study and may be useful in diagnostics of occupational voice disorders.
\end{abstract}

Key words:

Vocal acoustics and efficiency parameters, Software comparison

\section{INTRODUCTION}

The human voice, generated in the larynx due to the interaction of aerodynamic and acoustic mechanisms, is a complex phenomenon that requires a multifaceted approach and a comprehensive evaluation. That is why the human voice, like physical force, cannot be defined by only one measure [1-3].

Wiskirska-Woźnica et al. [4] remind that a simple relationship between the degree of acoustically assessed dysphonia and the extent of changes in the larynx does not exist. They

Received: January 18, 2012. Accepted: March 15, 2012.

Address reprint requests to J. Olszewski, Department of Otolaryngology and Laryngological Oncology, Medical University of Łódź, Żeromskiego 113, 90-549 Łódź, Poland (e-mail: jurek.olszewski@umed.lodz.pl). 
noticed that the majority of significant relationships between the study results could be found in the correlation assessment of parameters that define vocal folds vibrations in videostroboscopy and acoustic parameters of generated vocal wave in the Multi-Dimensional Voice Program (MDVP). Scientific and technological progress provides the ground for the application of more and more novel methods of voice assessment. However, this requires modern measurement devices and highly specialized staff able to perform and interpret various tests, although their value does not always meet the expectations of researchers [5,6].

The present methods for investigating phonatory activity of the larynx may be divided into six groups: laryngostroboscopy, assessment of the vocal folds vibration, acoustic techniques, aerodynamic tests, examination of vocal field and basic voice frequency, and laryngeal electromyography. In modern phoniatric or phonological laboratories acoustic analyses have become most common in the diagnosis of voice and speech disorders. The value of these tests has significantly increased due to the introduction of very fast digital voice analyzers, based on the latest computer generations with specially designed software [7,8].

In our Department the IRIS software has been used in voice acoustic assessment for a number of years [2,3], and now the DiagnoScope Specialist software is the newest tool for the sound perception assessment.

The aim of this study was to compare the parameters of vocal acoustic and efficiency analyses in medical students and academic teachers with use of the IRIS and DiagnoScope Specialist software and to evaluate their usefulness in prophylactic and preventive procedures and occupational disease certification which many a time is a difficult task [9].

\section{MATERIAL AND METHODS}

The study comprised 40 women (aged 22-39, mean: 23.5 years), including students and employees of the Military Medical Faculty, Medical University of Łódź.
After the informed consent of the study group had been obtained, a primary medical history was taken, videolaryngoscopic and stroboscopic examinations were performed and diagnostic vocal acoustic analysis was carried out with use of the IRIS and DiagnoScope Specialist software. The women under study were nonsmokers, drank alcohol occasionally and have been professional voice users for 3-15 years (mean: 7 years) in the communication process. No hormonal disorders were reported. No disorders were revealed on videolaryngoscopic and stroboscopic examinations.

The IRIS software module, a condenser microphone (Supercardioid XM1800S), Tube Ultragain MC100 devices and PC Realtek HD Audio sound cards were used for voice recording. Audio samples were recorded in a quiet room with a sound level of $30 \mathrm{~dB}$. In a short-term analysis, each woman under study was asked to phonate three times the sustained /a:/ vowel into the microphone placed $10 \mathrm{~cm}$ from her mouth.

After a few-minute break the voice was being recorded with use of the DiagnoScope Specialist software for the vocal acoustic and efficiency analyses and a computer condenser microphone.

In the diagnostic acoustic analysis, the sustained (approximately $1.5 \mathrm{~s}$ ) /a:/ vowel sound, produced twice on one breath, was recorded. The interval between the vowels, used to define the background noise level, was approximately $1 \mathrm{~s}$.

In the vocal efficiency analysis, the maximum prolonged phonation time of the sustained /a:/ vowel was recorded. The recording started when the phonation began (the moment found to be most convenient by the study participant). The physician started the recording as soon as the phonation was heard and stopped recording when it ceased.

Based on the recordings made with use of the IRIS and DiagnoScope Specialist software, the following vocal acoustic parameters were analyzed: relative average perturbation 
(Jitter, RAP), amplitude perturbation quotient (Shimmer, APQ), formant frequencies (F1, F2, F3, F4), harmonic perturbation quotient (HPQ), residual to harmonic (R2H), non-harmonic to harmonic (U2H), noise to harmonic ratio (NHR) and average fundamental frequency, $\mathrm{Hz}\left(\mathrm{F}_{0}\right)$.

The most important parameters in the module of vocal efficiency analysis, determined only with use of the DiagnoScope Specialist software, were:

- Phonation time - total length of all time intervals marked at the stage of the "analysis interval".

- True phonation time - total length of all basic periods within intervals denoted as those containing phonation, for which the value of the voiced parameter is not lower than the minimum value set at the stage of the "analysis interval".

- Efficiency coefficient (Perf Coef) - a numerical parameter dependent on voice quality expressed as the value of three short-term parameters (Jitter, $\mathrm{U} 2 \mathrm{H}$, and NHR) during the true phonation.

- Average efficiency (Average Perf) - efficiency coefficient divided by the true phonation time, the measure of average voice quality expressed as the value of three short-term parameters (Jitter, U2H, and NHR) within the total range of true phonation time. The results were statistically analyzed to evaluate minimum and maximum values and arithmetic mean of the chosen vocal parameters. Lin's concordance correlation coefficient of two measurement methods was applied to compare the values of average vocal acoustic parameters. A value of $p<0.001$ was considered to indicate statistical significance and in this case the compatibility between two methods/measurement devices.

\section{RESULTS}

Table 1 presents vocal acoustic parameters analyzed with use of the IRIS software; the range of their values are as follows:
- fundamental frequency $\mathrm{F}_{0}-186.67-306.64 \mathrm{~Hz}$ (mean: $249.36 \mathrm{~Hz}$ ); pitch perturbation quotient (Jitter) - 0.13-1.25 (mean: 0.39); RAP - 0.05-0.80 (mean: 0.24); relative amplitude perturbation (Shimmer) - 1.60-5.33 (mean: 3.14); APQ - 1.20-4.33 (mean: 2.44);

- formant values: F1 - 600.50-1093.63 (mean: 807.93); F2 - 1185.40-1718.60 (mean: 11433.99); F3 - 2335.30-3648.80 (mean: 2918.41); F4 - 3294.10-4309.60 (mean: 2918.42); HPQ - 4.67-12.67 (mean: 7.66); R2H - 12.00-36.67 (mean: 24.72); U2H - 1.33-3.73 (mean: 2.47); and NHR - 2.27-6.27 (mean: 4.11).

To compare vocal acoustic parameters obtained with use of the DiagnoScope Specialist software, the range of the following values is given:

- fundamental frequency $\mathrm{F}_{0}$, range $-188.00-322.00 \mathrm{~Hz}$ (mean: $247.97 \mathrm{~Hz}$; standard for women: $221.50 \mathrm{~Hz}$ ); pitch perturbation quotient (Jitter) - 0.22-1.40 (mean: 0.46; standard for women: 0.40); RAP - 0.120.85 (mean: 0.27; standard for women: 0.22); relative amplitude perturbation (Shimmer) - 2.10-5.80 (mean: 3.63; standard for women: 4.87); APQ - 1.404.40 (mean: 2.78; standard for women: 3.4);

- formant values: F1 - 367.00-975.00 (mean: 867.70; standard for women: 831.57); F2 - 1170.001751.00 (mean: 1413.05; standard for women: 1374.96); F3 - 2474.00-3355.00 (mean: 2875.62; standard for women: 2850.27); F4 - 3562.00-4358.00 (mean: 3935; standard for women: 3899.84); HPQ - 4.00-11.00 (mean: 7.73; standard for women: 9.13); R2H - 15.00-44.00 (mean: 22.92; standard for women: 29.3); U2H - 1.30-3.90 (mean: 2.39; standard for women: 3.0); and NHR - 2.20-6.80 (mean: 3.81; standard for women: 3.73)

The statistical analysis by means of Lin's concordance correlation coefficient showed the compatibility between two measurement software programs, IRIS and DiagnoScope 


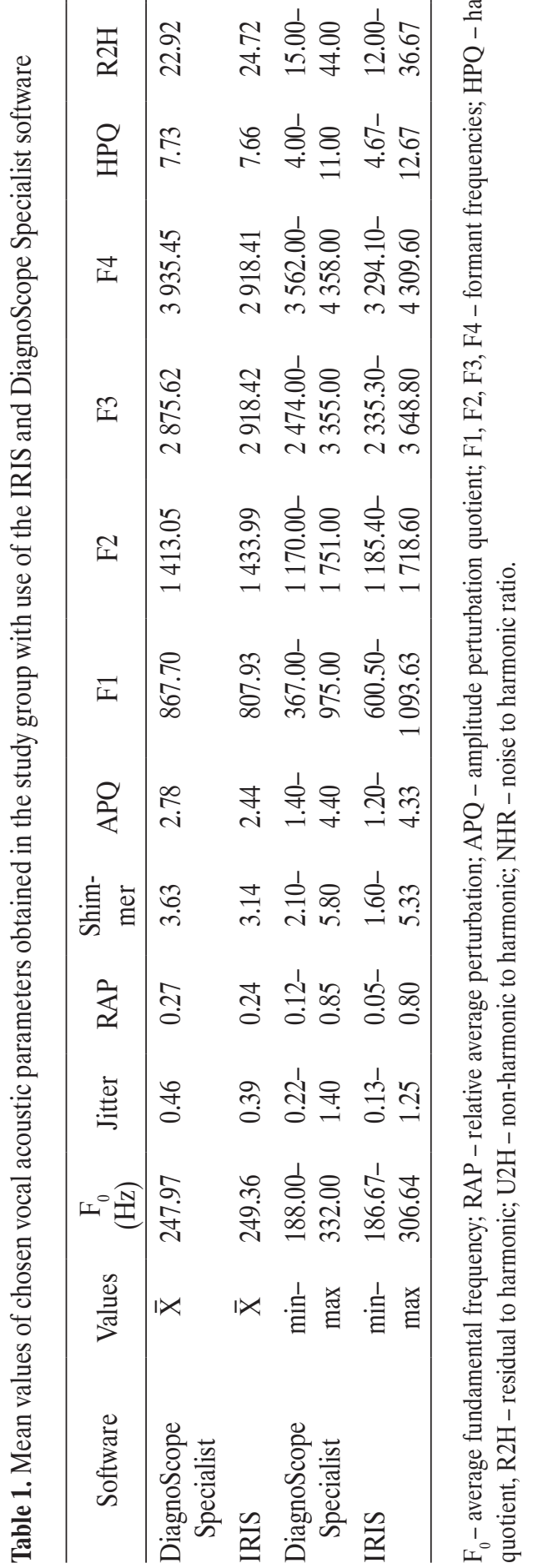

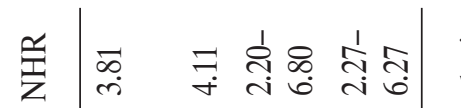

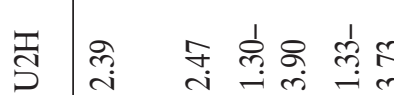

敢

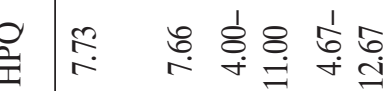

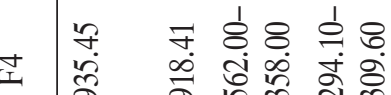

ข $\frac{1}{2} \circ \circ$

I

ㄴ.

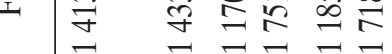

- ๙

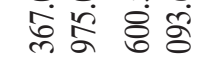

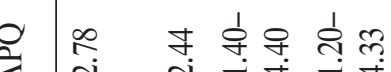

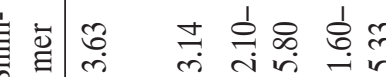

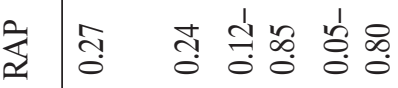

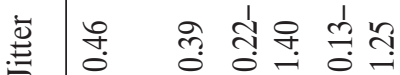

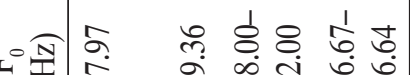

๖ $\backslash 1 \times 1$

온

证 戛

$\underbrace{}_{80} \frac{1}{2}$

苛

1.

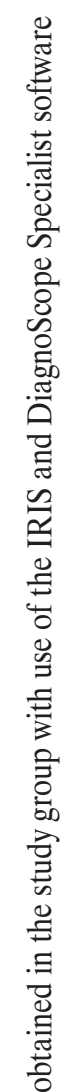

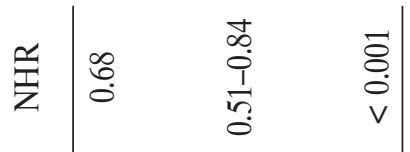

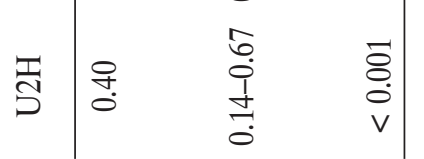

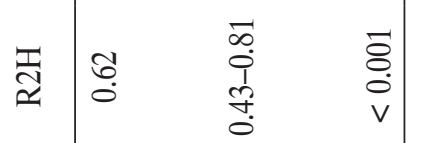

$\begin{array}{llll} & & 0 & \\ 0 & 0 & 0 \\ 0 & 0 & 0 \\ 0 & 0 & 0 \\ 0 & 0 & v\end{array}$

庄売乱

m

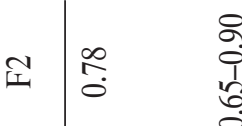

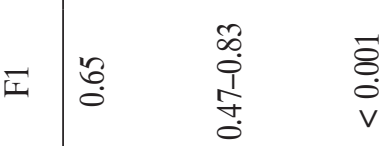

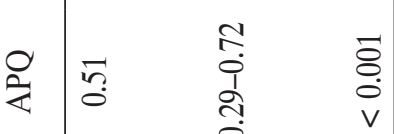

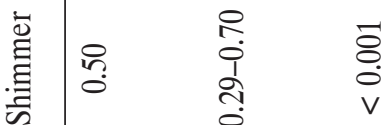

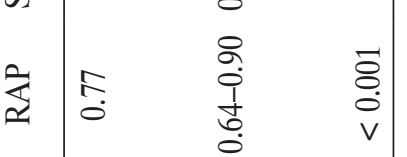

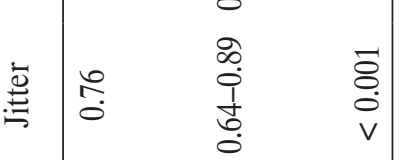

兽

芯

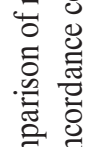

言苛

i.

음

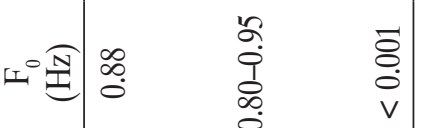

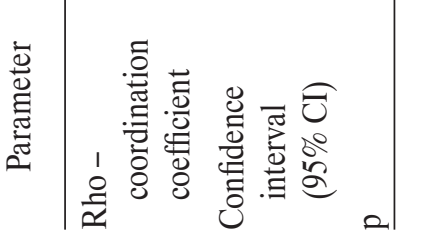

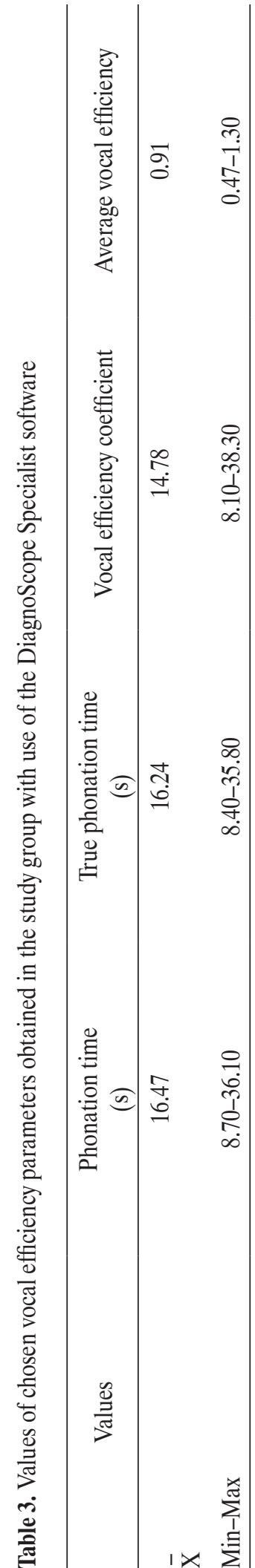


Specialist (statistically significant result) except for formant F4 (Table 2).

Vocal acoustic efficiency analysis was performed only with use of the DiagnoScope Specialist software (the analysis with use of the IRIS software was not feasible). The analyzed parameters showed the following range of the values:

- $\quad$ phonation time, range - 8.70-36.10 s (mean: $16.47 \mathrm{~s}$; standard for women: $21 \mathrm{~s}$ ); true phonation time 8.40-35.80 s (mean: $16.24 \mathrm{~s}$; standard for women: $21 \mathrm{~s})$;

- $\quad$ vocal efficiency coefficient - 8.10-38.30 (mean: 14.78; standard for women: 26.8); and average vocal efficiency - 0.47-1.30 (mean: 0.91; standard for women: 1.26) (Table 3).

\section{DISCUSSION}

In modern societies, about one third of the total labor force requires professional use of voice as a primary labor instrument [5]. It is estimated that in Poland about 900000 people are employed in the education sector. Bearing in mind that the number of non-public schools is still growing, this population is also increasing. The perspectives for the improved vocal health of teaching staff are rather positive. Awareness about the value of preventive actions has been changing among teachers. Pharmacological treatment and physiotherapy are not able to assure permanent recovery, therefore, the number of patients ready to participate in prophylactic and rehabilitation programs is still growing [10-12]. Updating and radical modification of a classical approach to the vocal fold structure have played a key role in the development of novel methods for laryngeal diagnostics.

The statistical analyses carried out under our study of vocal acoustic parameters evidenced the compatibility between two measurement software programs, IRIS and DiagnoScope Specialist, in objective voice assessment, except for only one parameter, namely formant F4.
The mean values of the parameters measured in medical students and academic teachers (videolaryngostroboscopy did show voice abnormalities) with use of IRIS software can be adopted as recognized standards for the female population as they have not as yet been worked out by the producer. To date, the values of the parameters obtained in a given patient before and after treatment could be only compared [2].

The measurement of voice acoustic parameters taken with use of DiagnoScope Specialist software showed some mean values higher or lower than standards given by the producer. Here are some examples of differences in values:

- fundamental frequency $\mathrm{F}_{0}-247.97 \mathrm{~Hz}$ (standard for women: $221.50 \mathrm{~Hz}$ ); pitch perturbation quotient (Jitter) - 0.46 (standard for women: 0.40 ); RAP - 0.27 (standard for women: 0.22); amplitude perturbation quotient (Shimmer) - 3.63 (standard for women: 4.87); APQ - 2.78 (standard for women: 3.4);

- formant values: F1 - 867.70 (standard for women: 831.57); F2 - 1413.05 (standard for women: 1374.96); F3 - 2875.62 (standard for women: 2850.25); F4 - 3935.45 (standard for women: 3899.84); HPQ - 7.73 (standard for women: 9.13); R2H - 22.92 (standard for women: 29.3); U2H - 2.39 (standard for women: 3.0); NHR - 3.81 (standard for women: 3.73).

Moreover, the IRIS software, unlike the DiagnoScope Specialist software, did not offer the option of performing the analysis of vocal acoustic efficiency. The mean values of the analyzed parameters were as follows: phonation time $-16.47 \mathrm{~s}$ (standard for women: $21 \mathrm{~s}$ ); true phonation time - $16.24 \mathrm{~s}$ (standard for women: $21 \mathrm{~s}$ ); vocal efficiency coefficient - 14.78 (standard for women: 26.8); and average vocal efficiency -0.91 (standard for women: 1.26).

Besides, laryngoscopy and laryngostroboscopy commonly used in everyday phoniatric practice, perceptual assessment of voice according to the GRBAS scale, 
recommended by the Japan Society of Logopedics and Phoniatrics, is an inexpensive and simple diagnostic method. The Voice Handicap Index (VHI) based on the selfassessment scale of voice disorders can be a useful tool to complete diagnostic procedures.

According to Obrębowski [7] the main challenge of social dimension facing phoniatrics is to elaborate principles of prophylaxis and prevention, applicable in practice and based on the objective evaluation, in order to prevent voice disorders in persons who occupationally strain their larynx and vocal folds.

\section{CONCLUSIONS}

1. The study evidenced the compatibility between two measurement software programs, IRIS and DiagnoScope Specialist, used to measure vocal acoustic parameters. Formant F4 is the only exception.

2. The advantage of the DiagnoScope Specialist software over the IRIS is that the DiagnoScop is provided with the standard values of vocal acoustic parameters developed by its producer, which are very close to our findings and can be useful in diagnostics of occupational voice disorders.

3. The analysis of vocal acoustic efficiency can be performed only with use of the DiagnoScope Specialist software.

\section{REFERENCES}

1. Obrębowski A, Świdziński P, Pruszewicz A, Świdziński T. Methods of larynx phoniatory function measurement in the Poznań University Department of Ear, Throat, Nose and Larynx Diseases between 1922 and 1939. Otolaryngol Pol 2007;61(5):719-23 [in Polish].

2. Kluch W, Olszewski J. Videolaryngostroboscopic examination of treatment effects in patients with chronic hyperthrophic larynx. Otolaryngol Pol 2008;62(6):680-5 [in Polish].
3. Kluch W, Olszewski J. The use of laryngostroboscopy in diagnostics and evaluation of treatment results in patients with organic lesions in the larynx. Otolaryngol Pol 2009;63(7): 11-5 [in Polish].

4. Wiskirska-Woźnica B, Pruszewicz A, Obrębowski A, Świdziński P. The correlation between subjective and objective voice assessment in organic and functional larynx disorders. Otolaryngol Pol 2003;(57)4:537-48 [in Polish].

5. Sinkiewicz A, Pawlak A, Wojnowski W, Owczarzak H. Assessment of rehabilitation of teachers' voice disorders. Otolaryngol Pol 2003;57(3):417-20 [in Polish].

6. Sinkiewicz A, Pruszewicz A, Obrębowski A, Wiskirska-Woźnica B, Wojnowski W. Subjective assessment of voice functions among teachers taking part in the rehabilitation program. Otolaryngol Pol 2006;60(3):391-5 [in Polish].

7. Obrębowski A. Communication disorders in the activity of the Department of Phoniatrics and Audiology, Medical University of Poznań. Otolaryngol Pol 2004;58(1):91-5 [in Polish].

8. Zielińska-Bliźniewska H, Kwaśniewska I, Miłoński J, Olszewski J. Evaluation of the vocal acoustic and efficiency analysis parameters in students with use of the new program DiagnoScope Specialist. Otolaryngol Pol 2011;65(3a):66-9 [in Polish].

9. Sułkowski WJ, Kowalska S. Occupational voice disorders: An analysis of diagnoses made and certificates issued in 1999-2004. Int J Occup Med Environ Health 2005;18(4):341-9.

10. Domeracka-Kołodziej A. Diseases of the larynx and voice disorders in teachers after many years of performing their profession, claiming the certification of occupational disease. Otolaryngol Pol 2001;55(6):671-2 [in Polish].

11. Ratajczak J, Rapiejko P, Jurkiewicz D. The effect of occupation on self-evaluation of voice disorders. Otolaryngol Pol 2007;61(5):857-63 [in Polish].

12. Orecka B, Namysłowski G, Fira R, Misiołek M, Ścierski W. Assessment of the larynx after microsurgery on the basis of videostroboscopy examination. Pol Merk Lek 2005;19(111):298300 [in Polish].

This work is available in Open Access model and licensed under a Creative Commons Attribution-NonCommercial 3.0 Poland License - http://creativecommons.org/ licenses/by-nc/3.0/pl/deed.en. 